OESOPHAGEAL DISEASE

\title{
Magnification chromoendoscopy for the detection of intestinal metaplasia and dysplasia in Barrett's oesophagus
}

\author{
P Sharma, A P Weston, M Topalovski, R Cherian, A Bhattacharyya, R E Sampliner
}

See end of article for authors' affiliations

.....................

Correspondence to: Dr P Sharma, Department of Veterans Affairs Medical Center, 4801 E Linwood Blvd, Kansas City, MO 64128-2295, USA psharma@kumc.edu

Accepted for publication 28 January 2002
Background: The presence of intestinal metaplasia (IM) in the columnar lined distal oesophagus defines Barrett's oesophagus with the risk of future malignant transformation. The distribution of both IM and dysplasia (low grade (LGD) and high grade (HGD)) within the columnar lined oesophagus is patchy and mosaic requiring random biopsies. Techniques that could help target areas of high yield within Barrett's mucosa would be helpful.

Aim: To study the utility of high magnification chromoendoscopy (MCE) in the detection of IM, LGD, and HGD in patients with Barrett's oesophagus.

Methods: Consecutive patients detected with columnar mucosa in the distal oesophagus were studied using an Olympus magnification endoscope (GIF-Q16OZ, 115x). The distal oesophagus was sprayed with indigo carmine solution and the oesophageal columnar mucosa patterns were noted under high magnification and targeted for biopsy. All biopsies were read by pathologists blinded to the endoscopic findings.

Results: Eighty patients with suspected Barrett's oesophagus (that is, columnar lined distal oesophagus) were studied: mean age 62.7 years (range 35-81). Mean length of columnar mucosa was $3.7 \mathrm{~cm}$ (range 0.5-17). Three types of mucosal patterns were noted within the columnar mucosa after spraying indigo carmine and using MCE: ridged/villous pattern, circular pattern, and irregular/distorted pattern. The yield of IM on target biopsies according to the patterns was: ridged/villous 57/62 (97\%) and circular 2/12 (17\%). Six patients had an irregular/distorted pattern and all had HGD on biopsy $(6 / 6(100 \%))$. Eighteen patients had LGD on target biopsies; all had the ridged/villous pattern. All patients with long segment Barrett's were identified using MCE whereas 23/28 patients (82\%) with short segment Barrett's had the ridged/villous pattern.

Conclusions: MCE helps visually identify areas with IM and HGD having specific patterns but not patients with LGD (appear similar to IM). MCE may be a useful clinical tool for the increased detection of patients with IM as well as for surveillance of patients for the detection of HGD. If these preliminary results are validated, MCE would help identify high yield areas, potentially eliminating the need for random biopsies.
B arrett's oesophagus is the premalignant lesion for adenocarcinoma of the oesophagus and oesophagogastric junction. It is a condition in which the squamous mucosa of the distal oesophagus is replaced by columnar mucosa, specifically intestinal metaplasia (IM). ${ }^{1}$ The incidence of oesophageal adenocarcinoma has been rapidly rising and has increased 3-4-fold over the past two decades. ${ }^{23}$ This has driven efforts to identify patients with the premalignant lesion-that is, Barrett's oesophagus. IM within the columnar lined oesophagus is the epithelial type that predisposes patients to the development of adenocarcinoma; the other two epithelial types are cardiac and fundic. ${ }^{4}$

As IM is part of the definition and is the epithelial type associated with cancer, obtaining biopsies from the columnar lined distal oesophagus is mandatory. ${ }^{5}$ The sensitivity and positive predictive values of standard upper endoscopy for diagnosing Barrett's oesophagus have been reported as $82 \%$ and $34 \%$, respectively. ${ }^{6}$ This is secondary to the patchy and mosaic presence of IM in the columnar distal oesophagus. Endoscopic screening is advocated in patients with chronic gastro-oesophageal reflux (GORD) symptoms for detection of Barrett's oesophagus with random biopsies being obtained if a columnar appearing distal oesophagus is visualised. ${ }^{7}$ Detection of IM from random biopsies of the columnar segment confirms the diagnosis of Barrett's oesophagus. These patients are subsequently enrolled in a surveillance programme.
Magnification endoscopy can provide high resolution mucosal detail of the gastrointestinal tissue and has been utilised in the colon and oesophagus. ${ }^{89}{ }^{10}$ Indigo carmine is a contrast stain which helps highlight the mucosal surface. ${ }^{8}$ Our goal was to utilise magnification endoscopy in combination with chromoscopy to help identify areas of IM and dysplasia within the columnar lined oesophagus.

\section{METHODS \\ Patients}

Patients undergoing upper endoscopy for chronic GORD symptoms, suspected Barrett's oesophagus, and those undergoing surveillance for Barrett's oesophagus were enrolled and gave written informed consent. Barrett's oesophagus was defined as the presence of columnar mucosa in the distal oesophagus of any length with IM on biopsy. ${ }^{11}$ The length of Barrett's, in patients with circumferential and non-circumferential segments, was measured from the

Abbreviations: GORD, gastro-oesophageal reflux disease; LGD, low grade dysplasia; HGD, high grade dysplasia; $M C E$, magnification chromoendoscopy; SSBO, short segment Barrett's oesophagus; LSBO, long segment Barrett's oesophagus; IM, intestinal metaplasia; LSS, light scattering spectroscopy. 
Table 1 Clinical feature of patients undergoing magnification chromoendoscopy

\begin{tabular}{ll}
\hline Total No & 80 \\
Mean age (range) (y) & 62.7 (35-81) \\
Sex (M/F) & $79 / 1$ \\
Ethnicity & 78 \\
$\quad$ Caucasian & 2 \\
$\quad$ African-American & $3.7(0.5-17)$ \\
Mean (range) Barrett's length (cm) & $39 / 41$ \\
LSBO/SSBO &
\end{tabular}

LSBO, long segment Barrett's oesophagus; SSBO, short segment Barrett's oesophagus.

gastro-oesophageal junction, defined as the most proximal portion of the gastric folds, ${ }^{12}$ to the proximally displaced squamocolumnar junction. Patient demographics, including age, sex, and ethnicity were noted and the length of Barrett's oesophagus was recorded at endoscopy.

\section{Magnification endoscope}

The magnification endoscope used in this study is an Olympus magnification endoscope, GIF-Q160Z, with a magnification of $115 \times$ (using a 14 inch television monitor). The outer diameter of the endoscope is $10.8 \mathrm{~mm}$. No special equipment, processing, or cleaning of the endoscope is required. This endoscope can function with either a CV-140 or CV-160 processor. A cap is fitted on the distal tip of the endoscope allowing the mucosa in contact with the cap to be magnified without the motility of the oesophagus affecting visualisation. Magnification is carried out using a lever located next to the up and down knob of the endoscope. When the lever is depressed fully, magnification of $115 \times$ is achieved.

\section{Magnification chromoendoscopy procedure}

At the time of upper endoscopy, the columnar mucosa in the distal oesophagus was sprayed with indigo carmine (0.4$0.8 \%$ ) using a spray catheter (Wilson Cook GT-7, Winston Salem, North Carolina, USA). Once the mucosa was exposed to the stain, the tip of the endoscope (with the cap in place) was brought into contact with the mucosa. The magnification lever was then fully depressed in order to achieve full magnification and the different mucosal patterns were carefully observed under magnification. At the initiation of the study, all investigators examined 4-6 magnification images of each pattern, agreed and formulated criteria for the different mucosal patterns which are described here. During the continuation of the study, each observer then applied these criteria for determining the different patterns during MCE. After a mucosal pattern was observed by the endoscopist, a biopsy forceps was then passed through the channel to the endoscope tip under magnification and the specific area, which had been just magnified, was then biopsied (radial jaw 3; 1599 Boston Scientific Corporation, Watertown, Massachusetts, USA).

\section{Histology}

All biopsy specimens were fixed in 10\% buffered formalin and submitted for histopathological examination. The tissues were then embedded in paraffin and stained with haematoxylineosin and alcian blue at $\mathrm{pH}$ 2.5. All biopsy specimens were then reviewed by pathologists who were blinded to the magnification endoscopy results and patterns. Dysplasia in the biopsies was classified as no dysplasia, low grade dysplasia (LGD), high grade dysplasia (HGD), and adenocarcinoma. ${ }^{13}$

\section{Statistical analysis}

The investigators examined the initial MCE images to decide on the various patterns visualised. Biopsy confirmed IM and dysplasia were used as the outcome measures. The sensitivity, specificity, and positive predictive value of MCE patterns for the detection of IM and dysplasia were then calculated.

\section{RESULTS}

Eighty patients with suspected Barrett's oesophagus were studied (table 1): mean age 62.7 years (range $35-81$ ). Mean length of columnar mucosa was $3.7 \mathrm{~cm}$ (range 0.5-17). Long segment Barrett's oesophagus (LSBO) was documented as $\geqslant 3$ $\mathrm{cm}$ of columnar mucosa whereas short segment Barrett's oesophagus (SSBO) was $<3 \mathrm{~cm}$ of columnar mucosa. All patients were receiving antisecretory therapy at the time of magnification endoscopy and there was no evidence of erosive oesophagitis. No nodules or plaques were visible on standard upper endoscopy, except in one patient, as described below. No adverse reaction to indigo carmine was identified in the group.

\section{MCE patterns}

Three types of mucosal patterns were noted within the columnar mucosa after spraying indigo carmine and using high magnification endoscopy: ridged/villous pattern, circular pattern, and irregular/distorted pattern (figs 1A, B, C).

- Ridge/villous pattern (fig 1A): presence of a clear villiform pattern including tortuous and thick villi, of sausage/ cerebrifom appearance but regular.

- Circular pattern (fig 1B): presence of circular and/or oval areas arranged in a regular fashion.

- Irregular/distorted pattern (fig lC): significant distortion and irregularity of the cerebriform and villous pattern.
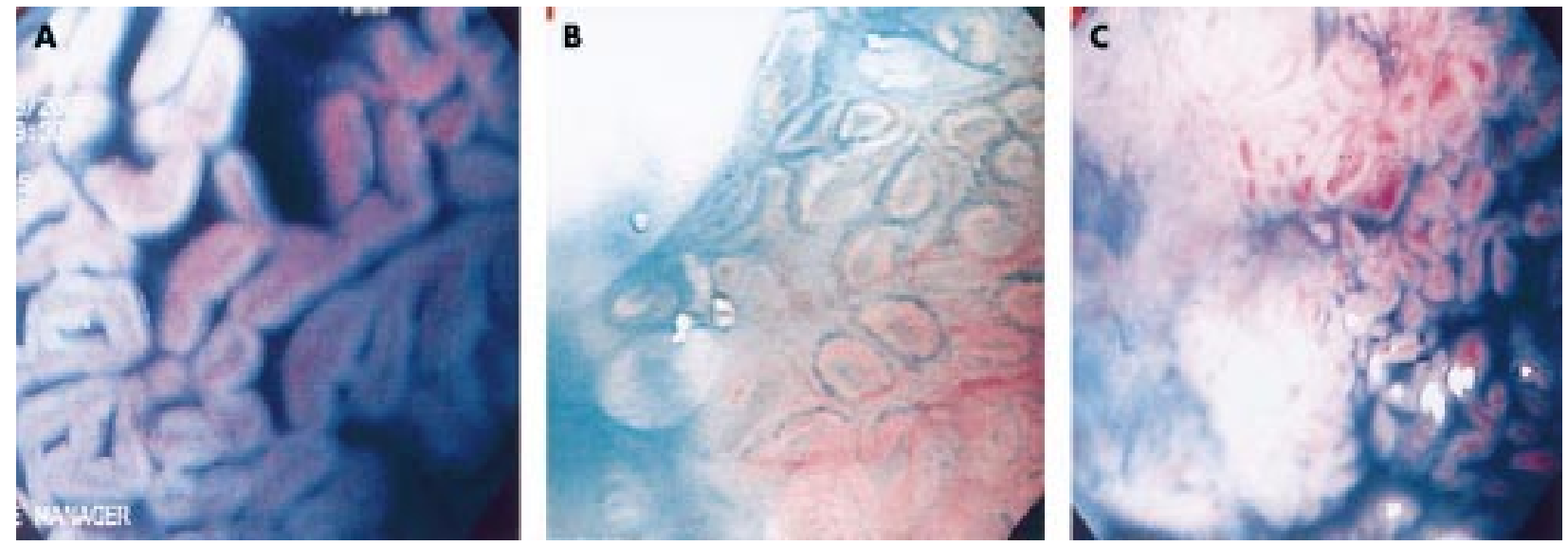

Figure 1 Three distinct mucosal patterns observed under magnification (115x) after spraying indigo carmine: ridge villous (A), circular (B), and irregular/distorted (C). 
Table 2 Patterns observed during magnification chromoendoscopy together with the yield for intestinal metaplasia and dysplasia

\begin{tabular}{lrllll}
\hline & $\mathrm{n}$ & $\begin{array}{l}\text { Patients with } \\
\text { suspected LSBO }\end{array}$ & $\begin{array}{l}\text { Patients with } \\
\text { suspected SSBO }\end{array}$ & $\begin{array}{l}\text { Detection } \\
\text { of IM }\end{array}$ & $\begin{array}{l}\text { Detection of } \\
\text { HGD }\end{array}$ \\
\hline Ridge/villous pattern & 62 & 34 & 28 & $97 \%$ & - \\
Circular pattern & 12 & 0 & 12 & $17 \%$ & - \\
Irregular/distorted pattern & 6 & 5 & 1 & - & $100 \%$ \\
\hline
\end{tabular}

LSBO, long segment Barrett's oesophagus; SSBO, short segment Barrett's oesophagus; IM, intestinal

metaplasia; HGD, high grade dysplasia.

The yield of IM on target biopsies according to the patterns was as follows: ridged/villous 57/62 (97\%) and circular 2/12 ( $17 \%)$. Thirty four of 80 patients had LSBO and all were identified using MCE (all had the ridge/villous pattern) whereas $23 / 28$ patients $(82 \%)$ with SSBO had the ridged/villous pattern (table 2). Sensitivity, specificity, and positive predictive values of the ridge/villous pattern for detecting IM were $97 \%$, $76 \%$, and $92 \%$, respectively. All patients with LSBO were detected with the ridge/villous pattern (sensitivity and specificity $100 \%$ ), and in the 41 patients with SSBO the sensitivity and specificity of the ridge/villous pattern for detecting IM were $92 \%$ and $69 \%$, respectively.

\section{Detection of dysplasia}

LGD was detected in 18 patients; all had the ridged/villous pattern. Six patients had an irregular/distorted pattern and all had HGD on biopsy $(6 / 6(100 \%))$ (table 2$)$. Mean length of Barrett's in patients with HGD was $7.6 \mathrm{~cm}$ (range 1.5-17); all were white males with a mean age of 72.5 years (range 67-80). One patient with a $5 \mathrm{~cm}$ long Barrett's oesophagus had a $2 \mathrm{~cm}$ mass (adenocarcinoma) within the Barrett's mucosa. However, other areas within the Barrett's were flat without any endoscopic lesions; MCE revealed an irregular/distorted pattern and biopsies showed HGD.

\section{DISCUSSION}

Currently, endoscopy with biopsy is the gold standard in making a diagnosis of Barrett's oesophagus. This however requires random biopsies which adds to the "hit and miss" nature and costs associated with the procedure. In a study of 570 patients undergoing upper endoscopy, Barrett's oesophagus was suspected in 146 patients; however, only 60 patients had a diagnosis confirmed by biopsy. ${ }^{6}$ SSBO was more frequently suspected than LSBO but was correctly diagnosed only $25 \%$ of the time compared with $55 \%$ for LSBO. The positive predictive value of endoscopy for making a diagnosis of Barrett's oesophagus was only $34 \%$. Thus improvements are clearly needed in improving our diagnostic techniques for this premalignant lesion.

In a relatively large series of patients undergoing upper endoscopy, our study has formulated objective MCE criteria that are useful in patients with suspected Barrett's oesophagus. The presence of the ridge/villous pattern had a high sensitivity, specificity, and positive predictive value-97\%, 76\%, and $92 \%$ respectively. This is in contrast with standard upper endoscopy, ${ }^{6}$ as reported earlier. The specificity of $76 \%$ could be due to potential biopsy targeting problems or interpretative errors of patterns (including the learning curve that may be present in this pilot study). MCE helps visually identify areas with IM and HGD that have specific patterns. However, this was not observed in areas with LGD which appeared similar to the IM pattern. This may be because the mucosal changes associated with LGD may not be significantly different than non-dysplastic areas (at the current magnification level) and also the high interobserver variability associated with the histological diagnosis of LGD. This is the first endoscopic study to identify HGD patterns distinct from IM using MCE in vivo. The procedure is similar to regular upper endoscopy; visualisation under magnification can be performed but no special light processors or additional instruments are required. The standard diameter of the endoscope allows it to be used as a regular diagnostic endoscope with the added benefit of using it for magnification purposes when required.

Two previous studies have utilised magnification endoscopy in patients with Barrett's oesophagus but with a maximum magnification of 35x. Stevens et al used Lugol's iodine or indigo carmine in combination with a $10-35 \times$ magnification endoscope and were able to detect IM in the 13 study patients. ${ }^{8}$ Recently, Guelrud et al evaluated 49 patients with short segment Barrett's oesophagus using acetic acid with magnification endoscopy $(35 \times)$. The yield of IM in areas with the villous and ridged pattern were $87 \%$ and $100 \%$, respectively. ${ }^{9}$ However, specific patterns for dysplasia have not been previously described.

A number of other newer endoscopic methods ${ }^{14-20}$ have also been tried for the optical detection of neoplasia, including light scattering spectroscopy (LSS), which uses reflected white light to provide morphological information (epithelial nuclear enlargement and crowding) about the target tissue. In a pilot study by Wallace et al, the sensitivity and specificity of LSS for detecting dysplasia, either LGD or HGD, were $90 \%$ and $90 \%$, respectively. ${ }^{15}$ Another technique, laser induced fluorescence spectroscopy, uses native tissue fluorophores that fluoresce in the ultraviolet and visible spectral regions-changes in fluorescence spectra caused by neoplastic tissue are analysed. Laser induced fluorescence has been found to be sensitive for the detection of diffuse HGD and adenocarcinoma but was less sensitive for the detection of focal HGD and LGD. ${ }^{16}$ Optical coherence tomography is yet another imaging technique that produces high resolution cross sectional images and in a pilot trial was found to be highly sensitive and specific for the detection of IM in the oesophagus and stomach. ${ }^{18}$ Vital dyes (chromoendoscopy) have also been used to identify IM and dysplasia within Barrett's oesophagus. Canto et al and Sharma et al have shown that methylene blue chromoscopy may enhance the detection of IM at endoscopy. ${ }^{21}{ }^{22}$ However, other centres have not had similar results. ${ }^{23}{ }^{24}$ Falk et al have also shown that a non-endoscopic method (brush cytology) can collect samples from the distal oesophagus ${ }^{25}$; the sensitivity for detecting HGD was $80 \%$ but only $25 \%$ for LGD and $18 \%$ for IM without dysplasia.

Magnification chromoendoscopy offers high resolution imaging. Our results show that MCE may be a significant improvement over standard endoscopy for diagnosing Barrett's. Possible future applications of this new technique include the recognition of IM in patients with suspected SSBO, and screening patients with chronic GORD for the documentation of Barrett's oesophagus as well as for the detection of HGD during surveillance endoscopy. The sensitivity and specificity results for detecting HGD were high but are based on a small number of patients and a larger number of such patients should be evaluated to confirm these results. This study is a significant first step towards establishing the diagnostic accuracy of MCE. These preliminary results need to be validated by different centres, and inter and intraobserver variability needs to be tested. The Barrett's surface area examined by this current method is small, which would make it 
very tedious to examine the entire surface area of longer segments of Barrett's. Also, observing the area under maximum magnification requires careful positioning of the endoscope which is helped by the cap at the endoscope tip. If these initial obstacles were overcome, MCE would be able to identify high yield areas, potentially eliminating the need for random biopsies. This may reduce sampling error and decrease/eliminate the need for biopsy that adds to the cost of the procedure. Further development of such technologies will enable us to visualise structures at the cellular and subcellular level, making it possible in the future to image goblet cells and/or dysplastic nuclei.

\section{Authors' affiliations}

P Sharma, A P Weston, M Topalovski, R Cherian, Department of Medicine, Gastroenterology, and Pathology Section, University of Kansas School Medicine and Veterans Affairs Medical Center, Kansas City, MO, USA

A Bhattacharyya, R E Sampliner, Department of Medicine, Gastroenterology, and Pathology Section, University of Arizona and Southern Arizona VA Healthcare System, Tucson, AZ, USA

Presented in part as an oral paper at the ASGE Topic Forum, Digestive Diseases Week, May 2001, Atlanta, GA.

\section{REFERENCES}

1 Weinstein WM, Ippoliti AF. The diagnosis of Barrett's esophagus: Goblets, goblets, goblets. Gastrointest Endosc 1996;44:91-5.

2 Blot WJ, Devesa SS, Kneller RW, et al. Rising incidence of adenocarcinoma of the esophagus and gastric cardia. JAMA 1991;265:1287-9

3 Devesa SS, Blot WJ, Fraumeni JF. Changing patterns in the incidence of esophageal and gastric carcinoma in the United States. Cancer 1988:83:2049-53.

4 Hamilton SR, Smith R, Cameron JL. Prevalence and characteristics of Barrett esophagus in patients with adenocarcinoma of the esophagus or esophagogastric junction. Hum Pathol 1988;19:942-8.

5 Sharma P. Endoscopic recognition and screening of Barrett's esophagus. Techniques Gastrointest Endosc 2000;2:182-5.

6 Eloubeidi MA, Provenzale D. Does this patient have Barrett's esophagus? The utility of predicting Barrett's esophagus at the index endoscopy. Am J Gastroenterol 1999;94:937-43.

7 Sampliner RE and the Practice Parameters Committee of the American College of Gastroenterology. Practice guidelines on the diagnosis, surveillance, and therapy of Barrett's esophagus. Am J Gastroenterol 1998;93:1028-32.
8 Stevens PD, Lightdale CJ, Green PHR, et al. Combined magnification endoscopy with chromoendoscopy for the evaluation of Barrett's esophagus. Gastrointest Endosc 1994;40:747-9.

9 Guelrud M, Herrera I, Essenfeld $\mathrm{H}$, et al. Enhanced magnification endoscopy: a new technique to identify specialized intestinal metaplasia in Barrett's esophagus. Gastrointest Endosc 2001;53:559-65.

10 Tada M, Kawai K. Research with the endoscope: new techniques using magnification and chromoendoscopy. Clin Gastroenterol 1986; 15:417-37

11 Sharma P, Morales TG, Sampliner RE. Short-segment Barrett's esophagus - The need for standardization of the definition and of endoscopic criteria. Am J Gastroenterol 1998;93:1033-6.

12 McClave SA, Boyce HW, Gottfried MR. Early diagnosis of columnar-lined esophagus: A new endoscopic criterion. Gastrointest Endosc 1987;33:413-16.

13 Haggitt RC. Barrett's esophagus, dysplasia, and adenocarcinoma. Hum Pathol 1994;25:982-93.

14 Panjehpour M, Overholt BF, Schmidhammer JL, et al. Spectroscopic diagnosis of esophageal cancer: new classification model, improved measurement system. Gastrointest Endosc 1995;41:577-81.

15 Wallace MB, Perelman LT, Bachman V, et al. Endoscopic detection of dysplasia in patients with Barrett's esophagus using light-scattering spectroscopy. Gastroenterology 2000;119:677-82.

16 Panjehpour M, Overholt BF, Vo-Dinh T, et al. Endoscopic fluorescence detection of high-grade dysplasia in Barrett's esophagus. Gastroenterology 1996;111:93-101.

17 Mayinger B, Horner $P$, Jordan $M$, et al. Light-induced autofluorescence spectroscopy for the endoscopic detection of esophageal cancer. Gastrointest Endosc 2001;54:195-201.

18 Poneros JM, Brand S, Bouma BE, et al. Diagnosis of specialized intestinal metaplasia by optical coherence tomography. Gastroenterology 2001;120:7-12.

19 Bouma BE, Tearney GJ, Compton CC, et al. High-resolution imaging of the human esophagus and stomach in vivo using optical coherence tomography. Gastrointest Endosc 2000;51:467-74.

20 Georgakoudi I, Jacobson BC, Van Dam J, et al. Flourescence, reflectance, and light-scattering spectroscopy for evaluating dysplasia in patients with Barrett's esophagus. Gastroenterology 2001;120:1620-9.

21 Canto MIF, Setrakian S, Willis J, et al. Methylene blue-directed biopsies improve detection of intestinal metaplasia and dysplasia in Barrett's esophagus. Gastrointest Endosc 2000;51:560-8.

22 Sharma P, Mayo M, Topalovski M, et al. Methylene blue chromoendoscopy for the detection of patients with short segment Barrett's esophagus. Gastrointest Endosc 2001;54:289-93.

23 Mukunda BR, Mayfield-Stokes S, Cecil B, et al. Results of the methylene blue-directed biopsy is similar to conventional biopsy for the diagnosis of intestinal metaplasia and dysplasia in Bararett's esophagus. Gastrointest Endosc 1999;49:AB54

24 Jobson B, Goenka P, Manalo G, et al. Methylene blue staining for intestinal metaplasia in Barrett's esophagus - is it as good as we think? Gastrointest Endosc 1999:49:AB52.

25 Falk GW, Chittajallu R, Goldblum JR, et al. Surveillance of patients with Barrett's esophagus for dysplasia and cancer with balloon cytology. Gastroenterology 1997;1 12:1787-97. 